\title{
SDF-1-CXCR4 axis: Cell trafficking in the cancer stem cell niche of head and neck squamous cell carcinoma
}

\author{
ANNE FABER, ULRICH REINHART GOESSLER, KARL HOERMANN, \\ JOHANNES DAVID SCHULTZ, CLAUDIA UMBREIT and JENS STERN-STRAETER \\ Department of Otorhinolaryngology Head and Neck Surgery, \\ University Medical Centre Mannheim, 68167 Mannheim, Germany
}

Received October 9, 2012; Accepted December 17, 2012

DOI: $10.3892 /$ or.2013.2380

\begin{abstract}
Stromal cell-derived factor-1 $\alpha$ (SDF-1 $\alpha$ ), also known as CXCL12, has variable effects on a plurality of cells. CXCR4 has been identified as its corresponding receptor. The SDF-1-CXCR4 axis is postulated to be a crucial key pathway in the interaction between (cancer) stem cells and their surrounding supportive cells in the cancer stem cell niche. We evaluated the expression of CD44 as a cancer stem cell marker and of CXCR4 in human HNSCC tissue samples. Afterwards, we monitored the concentration of SDF-1 in peripheral blood samples of HNSCC patients and healthy donors. We showed that CD44 and CXCR4 are expressed in human HNSCC tissues. Markedly, CD44 showed a high expression in HNSCC cells bordering cancer stromal cells. CXCR4 was mainly expressed in HNSCC tumor nests, but not in the surrounding stromal cells. No significant difference was noted between the SDF-1 concentration in the peripheral blood of HNSCC patients compared to healthy donors. We showed that CD44, as a stem cell marker in HNSCC, is located mainly at the borderline of HNSCC tumor nests with the surrounding cells. In addition, we demonstrated that CXCR4 as the corresponding receptor to SDF-1 is highly expressed in HNSCC tumor nests, but not in the tumor stroma. We collected evidence that SDF-1-CXCR4 interaction may be a crucial pathway in cell trafficking in the cancer stem cell niche of HNSCC, while SDF-1 was not detected in the peripheral blood of HNSCC patients. The SDF-1-CXCR4 axis may play an important role in the cancer stem cell theory of HNSCC. As SDF-1 $\alpha$ also exhibits a multitude of functional effects on HNSCC cells, such as migration and polarization, it may be
\end{abstract}

Correspondence to: Dr Anne Faber, Department of Otorhinolaryngology Head and Neck Surgery, University Medical Centre Mannheim, Theodor-Kutzer-Ufer 1-3, 68167 Mannheim, Germany

E-mail: aberanne@gmx.de

E-mail: anne.faber@umm.de

Abbreviations: HNSCC, head and neck squamous cell carcinoma; CSCs, cancer stem cells; SDF, stromal cell-derived factor

Key words: head and neck squamous cell carcinoma, cancer stem cells, stromal cell-derived factor, CXCR4, stem cell niche possible that the SDF-1-CXCR4 axis is also involved in the pathophysiology of the progression, recurrence and metastasis of malignant disease. Understanding these interactions may help to gain further insight into these mechanisms and as such help to discover new strategies of therapy.

\section{Introduction}

Although advances have been made in the surgical and non-surgical therapy of head and neck squamous carcinoma (HNSCC), the mortality rate from this disease has remained nearly constant over the last few years. This is mainly due to the development of therapy-resistant local and regional recurrences (1). Strategies of treatment apart from surgery, such as chemotherapy and radiation, eradicate a majority of proliferating cells in malignant tumors, but there is increasing evidence that there is a subpopulation of resistant tumor cells that cannot be reached by these regimens called cancer stem cells (CSCs) (2). As a result, it is imaginable that these cells are essential and responsible for initiation, but also maintenance and recurrence of malignant disease. CSCs have features of somatic stem cells such as self-renewal, differentiation and extensive proliferation ability. In recent years, the CSC hypothesis has also been assigned to $\operatorname{HNSCC}(3,4)$. Prince et al showed that $\mathrm{CD} 44^{+}$cells that were isolated out of the bulk of an HNSCC tumor, but not the CD44- cancer cells, gave rise to new tumors in a mouse model. These cells typically comprise $<10 \%$ of the cells in an HNSCC tumor (3). Other markers have been evaluated as cancer stem cell markers in HNSCC as well, such as ALDH1 (5) and CD133 (6). Current research aims to discover a combination of including and excluding surface markers to finally isolate the 'real CSC in HNSCC'.

CD44 is an integral cell membrane glycoprotein. It comprises different isoforms that arise from alternative splicing of a region of variable exons. Its apparent molecular mass ranges from 85 to $250 \mathrm{kDa}$, as its isoforms differ in the primary amino acid sequence and the amount of $\mathrm{N}$ - and O-glycosylation (7). At least 20 variants of CD44 have been reported due to the alternative splicing of 10 exons that encode the membrane's proximal portion of the extracellular domain (8-10).

In 1991, Günthert et al (11) and Hofmann et al (12) showed that the expression of CD44 gave metastatic potential to 
a non-metastatic cell line in a rat carcinoma model. Several analyses have indicated that there is a correlation between the expression of CD44 and progression, metastasis and prognosis of malignant disease and this has been shown in different types of epithelial malignancies, e.g. gastric cancer (13), hepatocellular carcinoma (14) or gynecological malignancies such as breast (15) or ovarian cancer (16). The in-depth analysis of expression markers such as CD44 in tissue samples of HNSCC patients may reveal their role as potential prognostic biomarkers or therapeutic targets, e.g. for antigen-directed immunotherapy.

The analysis of the cancer stem cell niche theory, where CSCs come into contact with their supportive cells surrounding them, may provide information concerning cell trafficking and underlying mechanisms, such as tumor expansion, recurrence and metastatic progression. The interaction between SDF-1 $\alpha$ and its receptor CXCR4 may play an important role in this field. SDF-1 $\alpha$ is a multifunctional cytokine that is expressed and secreted by several tissues, e.g. endothelium and stromal cells $(17,18)$, and is one component of the bulk of an HNSCC tumor. SDF-1 has a single open reading frame of 282 nucleotides encoding a polypeptide of 93 amino acids. It arises in two forms, SDF-1 $\alpha$ (amino acids 24-88) and SDF-1 $\beta$ (amino acids 24-93) by alternative splicing (18-20). SDF-1 $\alpha$ is the only proven chemoattractant for hematopoietic progenitor cells (HPCs) to date $(18,21-23)$. Thus, SDF-1 $\alpha$ is considered to be one of the key regulators for HPC trafficking between the peripheral circulation and the bone marrow $(17,18,23,24)$. Faber et al and others have demonstrated that SDF-1 $\alpha$ induces polarization and podia formation in HPCs and leukemic cells $(18,25)$, two properties that represent prerequisites for directed locomotion. SDF-1 $\alpha$ alone showed a moderate effect on cell proliferation in $\mathrm{CD}_{3} 4^{+}$ cells $(18,26)$, and its effect on survival or apoptosis of HPCs remains controversial (18,26-28). Furthermore, the SDF-1CXCR4 axis plays a crucial role in the regulation of homing and adhesion to the supportive cellular microenvironment in the hematopoietic stem cell niche (29). It remains unclear whether this interaction is also important in the cancer stem cell niche of malignant epithelial tumors such as HNSCC.

Signal transduction pathways initiated by the binding of SDF-1 $\alpha$ to CXCR4 are not fully understood. Mechanisms involved in CXCR4 signaling and downstream are multifaceted and include Gi-protein-mediated activation of intracellular components (30).

Herein, we monitored the expression of CD44 as a cancer stem cell marker and of CXCR4 as a potential target of interaction between CSCs and their supportive cells in the cancer stem cell niche in human HNSCC tissue samples. Accordingly, we evaluated SDF-1 serum levels in the peripheral blood of HNSCC patients compared to healthy donors to find evidence whether soluble interactions in the cancer stem cell niche of an HNSCC tumor can be detected in the periphery of human blood circulation. These findings may facilitate the development of therapeutic strategies particularly aimed at CSCs or particularly with regard to the SDF-1-CXCR4 axis aimed at the interaction of CSCs with their cellular cancer stem cell niche. Differences in the concentration of SDF-1 in the peripheral blood of HNSCC patients compared to healthy humans might lead to new strategies of tumor detection and the role of SDF-1 as a tumor marker.
Table I. HNSCC tissue collection.

\begin{tabular}{lccll}
\hline Patient & Gender & Age (yrs.) & Tumor location & TNM status \\
\hline 1 & M & 71 & Larynx & T4N0 \\
2 & M & 68 & Larynx & T3N2b \\
3 & M & 63 & Larynx & T4N0 \\
4 & M & NA & Oropharynx & T3N2c \\
5 & M & 67 & Oropharynx & T4N2M0 \\
6 & F & 50 & Oropharynx & T3N2c \\
7 & M & 76 & Hypopharynx & NA \\
8 & M & 59 & Hypopharynx & T4N2b \\
9 & F & 63 & Hypopharynx & T2N2b \\
\hline
\end{tabular}

$\mathrm{M}$, male; F, female; NA, information not available.

\section{Materials and methods}

Tissue and peripheral blood sample collection. A total of 9 HNSCC tissue samples from tumor patients was selected from a tissue database collected from 1997 to 2010 at the Department of Otorhinolaryngology, Head and Neck Surgery at the University of Mannheim. Samples were fixated immediately after excision by freezing in liquid nitrogen. All samples were confirmed by pathology after H\&E staining. The histological and clinical characteristics of all tumor samples are summarized in Table I.

In addition to the tissue collection, peripheral blood samples were obtained from HNSCC patients and healthy donors. Peripheral blood was collected before, during, but never after tumor surgery. A group of 11 healthy blood donors served as a control. Blood samples were centrifuged at $2500 \mathrm{rpm}$ for $10 \mathrm{~min}$. Afterwards, serum samples were harvested and fixated by freezing at $-80^{\circ} \mathrm{C}$. Characteristics of the peripheral blood samples are summarized in Table II.

Immunofluorescence labeling. To detect the expression of CD44 and CXCR4 in HNSCC tissue samples, tumors underwent fixation by freezing in liquid nitrogen as mentioned above. Specimens were sectioned $(5-\mu \mathrm{m})$, air-dried and fixated in acetone for $10 \mathrm{~min}$. Afterwards, the sections were treated with $4 \%$ paraformaldehyde (PFA) for $10 \mathrm{~min}$ at room temperature. After three washing steps with PBS, tumor samples were treated with $1 \%$ serum (goat) for another $10 \mathrm{~min}$. Sections were then incubated with the CD44/CXCR4 antibody (mouse monoclonal, 1:100; Abcam, Cambridge, UK) for $1 \mathrm{~h}$ at $37^{\circ} \mathrm{C}$ followed by incubation with a secondary biotinylated antibody (anti-mouse, 1:100) for $30 \mathrm{~min}$. After further washing steps with PBS, the sections were treated with Streptavidin-Cy3 (1:1000)/ Streptavidin-Alexia 488 (1:500) for $30 \mathrm{~min}$ at room temperature. Subsequently, sections were stained with DAPI after washing with PBS. Finally, slices were covered in FluorSave and dried to be evaluated by fluorescence microscopy.

Enzyme-linked immunosorbent assay. Serum levels of SDF were measured with a human SDF ELISA kit (R\&D Systems, 
Wiesbaden, Germany). A monoclonal antibody against soluble SDF was adsorbed to microwells in 96-well microtiter plates. Samples, including those with standards with known SDF concentrations, were pipetted into these wells. During the first incubation, the SDF antigen was added to the wells. After washing, a biotinylated monoclonal antibody specific for SDF was incubated, and the enzyme (streptavidin-peroxidase) was added. Following incubation and washing to remove all unbound enzymes, a substrate solution was added, which catalyzed a reaction on the bound enzyme and induced a colored reaction product. The intensity of this colored product is directly proportional to the concentration of SDF present in the samples.

Statistical analysis. All results are plotted as mean \pm standard deviation. To estimate the probability of differences, we adopted the Student t-test (two-tailed distribution, two-sample equal variance). A probability value $<0.05$ was considered to denote a statistically significant result.

\section{Results}

Tissue and peripheral blood sample collection. A total of 9 HNSCC tissue samples and the 29 blood serum samples were selected from a database collected from 1997 to 2010 at the Department of Otorhinolaryngology, Head and Neck Surgery at the Faculty of Medicine Mannheim, University of Heidelberg. Tissue samples used in the study were derived from 2 female and 7 male patients aged 50-76 years (mean age, 65 years). Blood samples were derived from 5 female and 24 male patients aged 48-76 years (mean age, 60 years). Sites of the primary tumors were the larynx, oropharynx, hypopharynx and oral cavity. Patient characteristics are summarized in Table I for tissue sample collection and in Table II for the blood sample collection. A group of 11 healthy blood donors, aged 26-87 years (mean age, 50 years) served as a control for experiments concerning the SDF concentration in peripheral blood of HNSCC patients.

Expression of CD44 in HNSCC tissues. Immunofluorescence labeling was performed to measure the expression of CD44 in HNSCC tissue samples via staining with Cy3. In HNSCC tissue samples, expression of CD44 was established in all cases. Its expression was mainly found in the cells forming the invasive front of the tumor. This invasive front is in direct contact with the tumor-surrounding stromal cells, and this interaction can be postulated as the cancer stem cell niche of HNSCC (Fig. 1A.4). Tumor samples stained in our experiments were mainly obtained from the superficial portions of the HNSCC tumors, although it was noted that parts located in the center of the tumor had a weaker staining pattern for CD44 (Fig. 1A.4). In general CD44 exhibited a membranous staining pattern (Figs. 1A.2 and A.3, and 2B.1). Nuclei were stained with DAPI.

Expression of CXCR4 in HNSCC tissues. Immunofluorescence labeling was performed to measure the expression of CXCR4 in HNSCC tissue samples by staining with Alexia 188. CXCR4 was present in all tissue samples stained. CXCR4 exhibited a surface staining pattern as well as cytoplasmic expression (Fig. 2B.2 and B.3). Specific expression of CXCR4
Table II. HNSCC patients enrolled for blood sample collection.

\begin{tabular}{|c|c|c|c|c|}
\hline Patient & Gender & Age (yrs.) & Tumor location & TNM \\
\hline 1 & M & 54 & Larynx & T4N2xMx \\
\hline 2 & $\mathrm{~F}$ & 55 & Larynx & T1N0 \\
\hline 3 & M & 62 & Larynx & T4N0 \\
\hline 4 & M & 70 & Larynx & T4N0 \\
\hline 5 & $\mathrm{M}$ & 68 & Larynx & $\mathrm{T} 3 \mathrm{~N} 2 \mathrm{~b}$ \\
\hline 6 & M & 68 & Larynx & NA \\
\hline 7 & M & 55 & Larynx & $\mathrm{T} 4 \mathrm{~N} 2 \mathrm{~b}$ \\
\hline 8 & M & 74 & Larynx & T4N0 \\
\hline 9 & M & 59 & Larynx & NA \\
\hline 10 & $\mathrm{M}$ & 57 & Larynx & T4N1 \\
\hline 11 & M & 55 & Oral cavity & T4N1 \\
\hline 12 & M & 69 & Oral cavity & $\mathrm{T} 1 \mathrm{~N} 2 \mathrm{~b}$ \\
\hline 13 & $\mathrm{~F}$ & 54 & Oral cavity & $\mathrm{T} 1 \mathrm{~N} 2 \mathrm{~b}$ \\
\hline 14 & $\mathrm{M}$ & 48 & Oral cavity & NA \\
\hline 15 & M & 49 & Oral cavity & T3N1 \\
\hline 16 & M & 51 & Oral cavity & NA \\
\hline 17 & M & 66 & Oral cavity & T2N0 \\
\hline 18 & $\mathrm{~F}$ & 50 & Oropharynx & $\mathrm{T} 3 \mathrm{~N} 2 \mathrm{c}$ \\
\hline 19 & $\mathrm{M}$ & 64 & Oropharynx & NA \\
\hline 20 & M & 60 & Oropharynx & $\mathrm{T} 4 \mathrm{~N} 2 \mathrm{c}$ \\
\hline 21 & $\mathrm{M}$ & 64 & Oropharynx & T3N2 \\
\hline 22 & $\mathrm{~F}$ & 55 & Oropharynx & $\mathrm{T} 4 \mathrm{~N} 3$ \\
\hline 23 & M & 61 & Hypopharynx & $\mathrm{T} 4 \mathrm{~N} 2 \mathrm{~b}$ \\
\hline 24 & $\mathrm{M}$ & 66 & Hypopharynx & T2N0 \\
\hline 25 & M & 76 & Hypopharynx & NA \\
\hline 26 & $\mathrm{M}$ & 59 & Hypopharynx & $\mathrm{T} 4 \mathrm{~N} 2 \mathrm{~b}$ \\
\hline 27 & $\mathrm{~F}$ & 62 & Hypopharynx & $\mathrm{T} 2 \mathrm{~N} 2 \mathrm{~b}$ \\
\hline 28 & M & 51 & Hypopharynx & NA \\
\hline 29 & $\mathrm{M}$ & 57 & Hypopharynx & T3N2c \\
\hline
\end{tabular}

M, male; F, female; NA, information not available.

at the invasive front of the tumor was not noted, while CXCR4 expression was high in the tumor nests, but was not noted in the tumor surrounding stroma (Fig. 2B.4). Thus, it was demonstrated that the corresponding receptor to SDF, which is transmitted by the supportive stromal cancer stem cell niche, can be found in the tumor nest as the counterpart of this interaction. Nuclei were stained with DAPI.

Concentration of SDF-1 in the peripheral blood of HNSCC patients. ELISA analysis was performed to evaluate the concentration of SDF-1 in the peripheral blood of HNSCC patients according to tumor site compared to a healthy control group. The was no significant difference in the SDF-1 concentration between tumor patients and the control group. Values are listed as means: control, $2057.91 \mathrm{pg} / \mathrm{ml}$; larynx, 2111.50 $\mathrm{pg} / \mathrm{ml}$; oral cavity, $1891.43 \mathrm{pg} / \mathrm{ml}$; oropharynx, $2244.2 \mathrm{pg} / \mathrm{ml}$; hypopharynx, $2000.29 \mathrm{pg} / \mathrm{ml}$. 

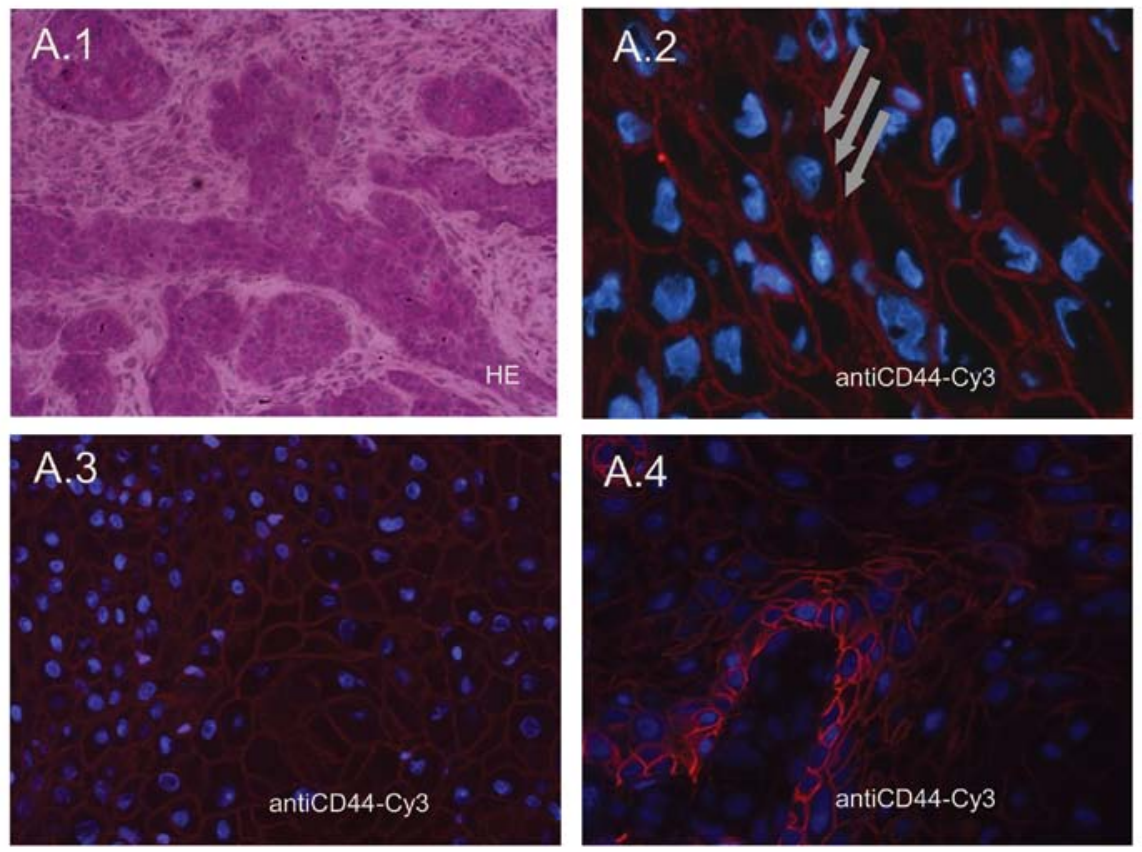

Figure 1. Expression of CD44 in HNSCC. Identification of the cancer stem cell niche. After verification of pathology by H\&E staining (A.1), immunofluorescence labeling was performed to measure expression of CD44 in HNSCC tissue samples via staining with Cy3 (A.2-4). Arrows indicate the expression of CD44 marked red by $\mathrm{Cy} 3$. In the HNSCC tissue samples stained, expression of CD44 was established and was mainly noted in the cells forming the invasive front of the tumor (A.4). Nuclei were stained with DAPI.
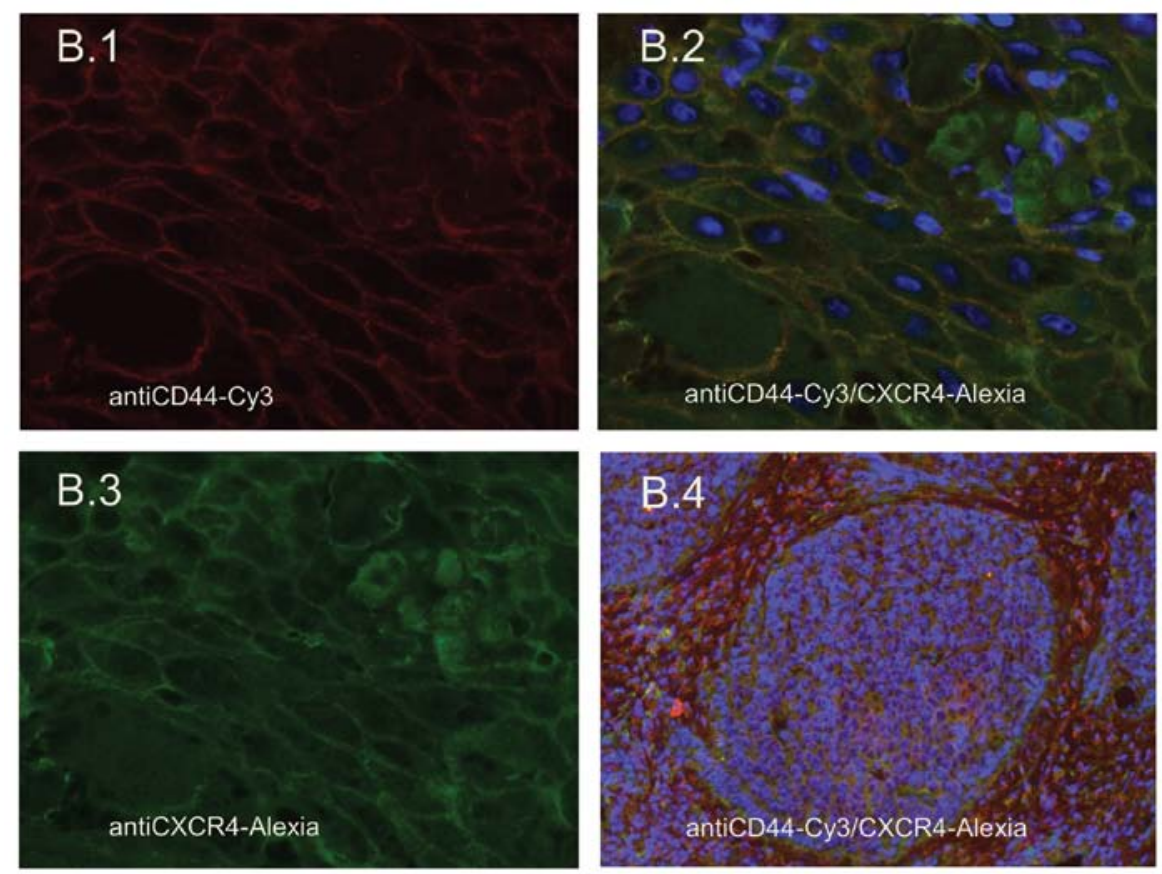

Figure 2. Expression of CD44 and CXCR4 in HNSCC tissue samples. Interactions in the cancer stem cell niche. CD44 exhibited a membranous staining pattern (red in B.1). CXCR4 was also expressed in the cytoplasm (green in B.3). CXCR4 was mainly expressed in the tumor nests, but not in the supportive cells surrounding the tumor (green in B.4). Nuclei were stained with DAPI.

\section{Discussion}

In the past few years, the stem cell theory has become more important in the field of tumor biology, particularly in issues concerning tumor development, progression and metastasis. The cancer stem cell theory has generated new ideas and discussion in the research of malignant disease and in therapeutic options. At present, the presence of cancer stem cells are not only known to exist in hematological malignancies but also in solid tumor entities $(3,31)$. The cancer stem cell theory in solid tumors such as HNSCC has had dramatic consequences. To date, therapeutic interventions, e.g. surgery 
or chemoradiation, are directed towards the bulk of the tumor without focusing on a small amount of specialized tumor cells which have the facilities of self-renewal, differentiation and unlimited proliferation. The question of which combination of markers should be used to isolate 'cancer stem cells' e.g. in HNSCC remains unclear. In addition, the combination of markers appears to vary in different types of tumors $(3,31,32)$. In the hematopoietic system, CD34 has been shown to play an important role in this context (18), and in acute myeloid leukemia, $\mathrm{CD} 34^{+}$cells were able to establish a new tumor in a mouse model (33). Regarding solid tumor entities, CD133 has been postulated to play an important role in malignancies of the nervous system, and results showed that $\mathrm{CD}_{133^{+}}$cells are relatively radioresistant (34) which can also be assessed as a feature of cancer stem cells. In contrast, CD133 is a ubiquitous marker in the hematopoietic and endothelial progenitor cell system (35), and it has also been shown to be present in the neurologic system, e.g. in glioblastoma (36).

In HNSCC, the presence of cancer stem cells has also been postulated. Prince et al showed that $\mathrm{CD} 44^{+}$cells, compared to CD44- cells, were able to engraft an entire new HNSCC tumor in a mouse model (3). According to their results, the cell selection for $\mathrm{CD}_{4} 4^{+}$was sufficient to isolate cells with cancer stem cell properties out of the bulk of an HNSCC tumor (3). Unfortunately, CD44 is also expressed in ordinary cells (35). The prevalent challenge of research is to identify a combination of surface markers that can identify the subgroup of potent CSCs from the bulk of a tumor more precisely. CD44 cannot be sufficiently used as a defining CSC marker alone, since as a cell surface glycoprotein it takes part in numerous cell-cell interactions $(35,37)$ and in humans it is present in a multitude of splice variants (35) and can be found ubiquitously. Approaches to the investigations of the 'real cancer stem cell' in HNSCC requires research with human tumor material with isolation of a small subpopulation of cells out of the bulk of the tumor using complicated cell separation steps including recurrent washing steps, lysis by DNAses and FACS-sorting. To concur with the current standard of knowledge, a combination of lineage markers must be negative to separate CSCs out of the entire HNSCC tumor (CD3, CD3, CD10, CD18, CD31, CD64, CD140) $(3,35,38)$. Other CSC markers (CD44, ALDH1) have been proposed, but only small amounts of cells carrying these markers are said to be able to initiate an HNSCC tumor in a mouse model $(3,5)$. The cell amount remaining after this separation as described above is often minute and the remaining cells are often not useful for further experiments. Often it is necessary to revert to cell lines to perform experiments. ALDH1 is another marker postulated as a cancer stem cell marker in HNSCC (5). It was also postulated as a cancer stem cell marker in breast (39) and lung cancer (40). It was shown that high expression of ALDH1 in breast cancer is associated with poor prognosis and early metastasis (41). It is an enzyme that catalyzes the oxidation of aromatic aldehydes into carboxylic acids and it has been shown to be responsible for the resistance of progenitor cells to chemotherapeutic agents by breaking down cytotoxic drugs $(35,42)$.

In a present study concerning HNSCC, ALDH1 was declared to be a more specific cancer stem cell marker in comparison to CD44 (5). In the present study, only CD44 was used as a cancer stem cell marker, and we found a high expression of CD44 in all tissue samples tested especially at the invasive front of the tumor, where HNSCC cells come into contact with their surrounding cells, e.g. stromal cells. Our findings corroborate Sterz et al (43) who showed colocalization of CD44, ALDH1 and CK14 in the basal layer of HNSCC and at the border of the tumor at the tumor-stroma border. The localization of cancer stem cell candidates at the border of tumors is feasible as this is where invasion and the main tumor growth occurs. Highly differentiated cell layers were negative for these markers, and this agrees with the supposition that stem cells are localized close to the basal membrane. Invasiveness and metastasis of tumors also depend on their capacity to penetrate and rebuild the extracellular matrix (44). Malignant cells infiltrate healthy tissue by degradation of the extracellular matrix components, by breaking down vessel borders and by promoting metastasis in distant organs. It has been shown for different tumor entities, that the presence of matrix metalloproteinases plays an important role in this process and this has also been shown for HNSCC $(43,45)$. In HNSCC, MMP-9 and -2 have been shown by Sterz et al to be located at the invasive front of HNSCC (43).

The SDF-CXCR4 axis is involved in several aspects of tumor progression, such as angiogenesis, metastasis and survival (46). The microenvironment of the bone marrow has been reported to support survival, differentiation and proliferation of hematopoietic progenitor cells (47), but also malignant progenitor cells of the hematopoietic system, e.g. B-cell acute lymphoblastic leukemia (B-ALL) (48). The pathway which includes the SDF-1-CXCR4 axis has been postulated to be responsible for retention of lymphoid and myeloid leukemia cells in the bone marrow $(48,49)$. It becomes obvious that the importance of the SDF-1-CXCR4 axis in the hematopoietic system is well-discussed, but this is the first time that the SDF-1-CXCR4 axis has been reported to play a crucial role in the development and the progression of invasion and metastasis of HNSCC.

In this study, we showed that CXCR4 can be found in tumor nests of HNSCC, but not in the surrounding stromal region of the cancer stem cell niche. It has been shown by Clatot et al that the intratumoral level of SDF-1 is correlated with survival in HNSCC (50). In contrast, we showed in this study that the concentration of SDF-1 in the peripheral blood of HNSCC patients does not differ in comparison to healthy donors. This suggests that the SDF-1-CXCR4 axis plays a role in the cancer stem cell niche within the tumor, but not in the periphery of the blood system. In previous studies, we showed that polarization and formation of filopodia and a prominent uropod were increased in the $\mathrm{CD} 44^{+} \mathrm{CXCR} 4^{+}$ HNSCC cell line UM-SCC 11A in a dose-dependent manner by SDF-1 $\alpha$. This effect can probably be attributed to cytoskeleton rearrangements of actin-containing protrusions $(18,51)$, and this also might be influenced by extracellular factors such as matrix metalloproteinases $(51,52)$. Podia formation was found to occur together with cell adhesion especially to the microenvironment (18). If evidence can be found that podia formation and adhesion to the cellular cancer stem cell niche are also associated with HNSCC CD44+ cells, understanding of these interactions would offer insight into new strategies of cancer-directed therapy in HNSCC. For example small-molecule agonists or antagonists of SDF-1 may be 
used to interfere with the cancer stem cell niche resulting in inhibition or ideally blockage of tumor invasion and metastasis (18). Further experiments using human material are warranted to expand and specify our insight regarding the cell-cell interactions in the cancer stem cell niche of solid tumors. Based on the findings it may be possible to develop particular therapeutic strategies aimed at CSCs or the SDF-1CXCR4 axis.

\section{Acknowledgements}

We gratefully thank Petra Prohaska for the excellent technical support.

\section{References}

1. Boehm A, Wichmann G, Mozet C and Dietz A: Current therapy options in recurrent head and neck cancer. HNO 58: 762-769, 2010 (In German)

2. Mannelli G and Gallo O: Cancer stem cells hypothesis and stem cells in head and neck cancers. Cancer Treat Rev 38: 515-539, 2012.

3. Prince ME, Sivanandan R, Kaczorowski A, et al: Identification of a subpopulation of cells with cancer stem cell properties in head and neck squamous cell carcinoma. Proc Natl Acad Sci USA 104: 973-978, 2007.

4. Oker N, Kaufmann AM and Albers AE: Biology and relevance of stem cells in squamous head and neck cancer: latest insights and review of literature. Laryngorhinootologie 91: 326-332, 2012 (In German).

5. Clay MR, Tabor M, Owen JH, et al: Single-marker identification of head and neck squamous cell carcinoma cancer stem cells with aldehyde dehydrogenase. Head Neck 32: 1195-1201, 2010.

6. Chen YS, Wu MJ, Huang CY, et al: CD133/Src axis mediates tumor initiating property and epithelial-mesenchymal transition of head and neck cancer. PLoS One 6: e28053, 2011.

7. Franzmann EJ, Reategui EP, Pedroso F, et al: Soluble CD44 is a potential marker for the early detection of head and neck cancer. Cancer Epidemiol Biomarkers Prev 16: 1348-1355, 2007.

8. Ponta H, Wainwright D and Herrlich P: The CD44 protein family. Int J Biochem Cell Biol 30: 299-305, 1998.

9. Screaton GR, Bell MV, Bell JI and Jackson DG: The identification of a new alternative exon with highly restricted tissue expression in transcripts encoding the mouse Pgp-1 (CD44) homing receptor. Comparison of all 10 variable exons between mouse, human, and rat. J Biol Chem 268: 12235-12238, 1993

10. Screaton GR, Bell MV, Jackson DG, Cornelis FB, Gerth U and Bell JI: Genomic structure of DNA encoding the lymphocyte homing receptor CD44 reveals at least 12 alternatively spliced exons. Proc Natl Acad Sci USA 89: 12160-12164, 1992.

11. Günthert U, Hofmann M, Rudy W, et al: A new variant of glycoprotein CD44 confers metastatic potential to rat carcinoma cells. Cell 65: 13-24, 1991.

12. Hofmann M, Rudy W, Zoller M, et al: CD44 splice variants confer metastatic behavior in rats: homologous sequences are expressed in human tumor cell lines. Cancer Res 51: 5292-5297, 1991.

13. Zhang H, Xi H, Cai A, et al: Not all side population cells contain cancer stem-like cells in human gastric cancer cell lines. Dig Dis Sci: Aug. 10, 2012 (Epub ahead of print).

14. Pang RW and Poon RT: Cancer stem cell as a potential therapeutic target in hepatocellular carcinoma. Curr Cancer Drug Targets: Aug. 2, 2012 (Epub ahead of print).

15. Park SY, Lee HE, Li H, Shipitsin M, Gelman R and Polyak K: Heterogeneity for stem cell-related markers according to tumor subtype and histologic stage in breast cancer. Clin Cancer Res 16: 876-887, 2010.

16. Zeimet AG, Reimer D, Sopper S, et al: Ovarian cancer stem cells. Neoplasma 59: 747-755, 2012.

17. Dar A, Goichberg P, Shinder V, et al: Chemokine receptor CXCR4-dependent internalization and resecretion of functional chemokine SDF-1 by bone marrow endothelial and stromal cells. Nat Immunol 6: 1038-1046, 2005.
18. Faber A, Roderburg C, Wein F, et al: The many facets of SDF-1alpha, CXCR4 agonists and antagonists on hematopoietic progenitor cells. J Biomed Biotechnol 2007: 26065, 2007.

19. De La Luz Sierra M, Yang F, Narazaki M, et al: Differential processing of stromal-derived factor-1alpha and stromal-derived factor-1beta explains functional diversity. Blood 103: 2452-2459, 2004.

20. Shirozu M, Nakano T, Inazawa J, et al: Structure and chromosomal localization of the human stromal cell-derived factor 1 (SDF1) gene. Genomics 28: 495-500, 1995.

21. Kim $\mathrm{CH}$ and Broxmeyer HE: In vitro behavior of hematopoietic progenitor cells under the influence of chemoattractants: stromal cell-derived factor-1, steel factor, and the bone marrow environment. Blood 91: 100-110, 1998 .

22. Mohle R, Bautz F, Rafii S, Moore MA, Brugger W and Kanz L: The chemokine receptor CXCR-4 is expressed on CD34 ${ }^{+}$ hematopoietic progenitors and leukemic cells and mediates transendothelial migration induced by stromal cell-derived factor-1. Blood 91: 4523-4530, 1998

23. Petit I, Goichberg P, Spiegel A, et al: Atypical PKC-zeta regulates SDF-1-mediated migration and development of human CD34 ${ }^{+}$ progenitor cells. J Clin Invest 115: 168-176, 2005.

24. Chute JP: Stem cell homing. Curr Opin Hematol 13: 399-406, 2006.

25. Fruehauf S, Srbic K, Seggewiss R, Topaly J and Ho AD: Functional characterization of podia formation in normal and malignant hematopoietic cells. J Leukoc Biol 71: 425-432, 2002.

26. Lataillade JJ, Clay D, Dupuy C, et al: Chemokine SDF-1 enhances circulating CD34(+) cell proliferation in synergy with cytokines: possible role in progenitor survival. Blood 95: 756-768, 2000.

27. Broxmeyer HE, Kohli L, Kim CH, et al: Stromal cell-derived factor-1/CXCL12 directly enhances survival/antiapoptosis of myeloid progenitor cells through CXCR4 and G(alpha)i proteins and enhances engraftment of competitive, repopulating stem cells. J Leukoc Biol 73: 630-638, 2003.

28. Kijowski J, Baj-Krzyworzeka M, Majka M, et al: The SDF-1CXCR4 axis stimulates VEGF secretion and activates integrins but does not affect proliferation and survival in lymphohematopoietic cells. Stem Cells 19: 453-466, 2001.

29. Hattori K, Heissig B, Tashiro K, et al: Plasma elevation of stromal cell-derived factor-1 induces mobilization of mature and immature hematopoietic progenitor and stem cells. Blood 97: 3354-3360, 2001.

30. Kremer KN, Clift IC, Miamen AG, et al: Stromal cell-derived factor-1 signaling via the CXCR4-TCR heterodimer requires phospholipase C- $\beta 3$ and phospholipase $\mathrm{C}-\gamma 1$ for distinct cellular responses. J Immunol 187: 1440-1447, 2011.

31. La Porta CA: Thoughts about cancer stem cells in solid tumors. World J Stem Cells 4: 17-20, 2012.

32. Grosse-Gehling P, Fargeas CA, Dittfeld C, et al: CD133 as a biomarker for putative cancer stem cells in solid tumours: limitations, problems and challenges. J Pathol: Aug. 16, 2012 (Epub ahead of print).

33. Schubert M, Herbert N, Taubert I, et al: Differential survival of AML subpopulations in NOD/SCID mice. Exp Hematol 39: 250-263, 2011.

34. Jamal M, Rath BH, Tsang PS, Camphausen $\mathrm{K}$ and Tofilon PJ: The brain microenvironment preferentially enhances the radioresistance of CD133(+) glioblastoma stem-like cells. Neoplasia 14: 150-158, 2012.

35. Wollenberg B: Implication of stem cells in the biology and therapy of head and neck cancer. Laryngorhinootologie 90 (Suppl 1): S110-S119, 2011 (In German).

36. Warrier S, Pavanram P, Raina D and Arvind M: Study of chemoresistant $\mathrm{CD}_{133^{+}}$cancer stem cells from human glioblastoma cell line U138MG using multiple assays. Cell Biol Int: Jul. 6, 2012 (Epub ahead of print).

37. Jaggupilli A and Elkord E: Significance of CD44 and CD24 as cancer stem cell markers: an enduring ambiguity. Clin Dev Immunol 2012: 708036, 2012.

38. Pries R, Wittkopf N, Hasselbacher K and Wollenberg B: Constitutive expression of the potential stem cell marker CD44 in permanent HNSCC cell lines. HNO 56: 461-466, 2008 (In German).

39. Badve $\mathrm{S}$ and Nakshatri H: Breast-cancer stem cells - beyond semantics. Lancet Oncol 13: e43-e48, 2012.

40. Jiang F, Qiu Q, Khanna A, et al: Aldehyde dehydrogenase 1 is a tumor stem cell-associated marker in lung cancer. Mol Cancer Res 7: 330-338, 2009. 
41. Charafe-Jauffret E, Ginestier C, Iovino F, et al: Aldehyde dehydrogenase 1-positive cancer stem cells mediate metastasis and poor clinical outcome in inflammatory breast cancer. Clin Cancer Res 16: 45-55, 2012.

42. Silva IA, Bai S, McLean K, et al: Aldehyde dehydrogenase in combination with CD133 defines angiogenic ovarian cancer stem cells that portend poor patient survival. Cancer Res 71: 3991-4001, 2011.

43. Sterz CM, Kulle C, Dakic B, et al: A basal-cell-like compartment in head and neck squamous cell carcinomas represents the invasive front of the tumor and is expressing MMP-9. Oral Oncol 46: 116-122, 2010

44. Nakajima M, Gohji K, Fabra A, Fidler IA and Tsuruo T: Regulation of tumor metastasis and extracellular matrix degradative enzyme production by microenvironments. Gan To Kagaku Ryoho 20: 380-386, 1993 (In Japanese).

45. Schultz JD, Rotunno S, Erben P, et al: Down-regulation of MMP-2 expression due to inhibition of receptor tyrosine kinases by imatinib and carboplatin in HNSCC. Oncol Rep 25: 1145-1151, 2011.

46. Teicher BA and Fricker SP: CXCL12 (SDF-1)/CXCR4 pathway in cancer. Clin Cancer Res 16: 2927-2931, 2010.
47. Metzen E, Jin F, Brockmeier U and Otterbach F: New insight into the SDF-1/CXCR4 axis in a breast carcinoma model: hypoxiainduced endothelial SDF-1 and tumor cell CXCR4 are required for tumor cell intravasation. Mol Cancer Res 10: 1021-1031, 2012.

48. Purizaca J, Meza I and Pelayo R: Early lymphoid development and microenvironmental cues in B-cell acute lymphoblastic leukemia. Arch Med Res 43: 89-101, 2012.

49. Juarez J, Dela Pena A, Baraz R, et al: CXCR4 antagonists mobilize childhood acute lymphoblastic leukemia cells into the peripheral blood and inhibit engraftment. Leukemia 21: 1249-1257, 2007.

50. Clatot F, Picquenot JM, Choussy O, et al: Intratumoural level of SDF-1 correlates with survival in head and neck squamous cell carcinoma. Oral Oncol 47: 1062-1068, 2011.

51. Geutskens SB, Andrews WD, van Stalborch AM, et al: Control of human hematopoietic stem/progenitor cell migration by the extracellular matrix protein Slit3. Lab Invest 92: 1129-1139, 2012.

52. Ghosh MC, Makena PS, Gorantla V, Sinclair SE and Waters CM: CXCR4 regulates migration of lung alveolar epithelial cells through activation of Rac1 and matrix metalloproteinase-2. Am J Physiol Lung Cell Mol Physiol 302: L846-L856, 2012. 